\title{
Introduction to Cognitive Neuroscience and Psychophysiology in the Digital Economy Minitrack
}

\author{
Randall Minas \\ University of Hawaii at Manoa \\ rminas@hawaii.edu
}

Individual cognition plays an integral role in our interactions with the Internet and the Digital Economy. Over the past decade, tools from psychophysiology and cognitive neuroscience have entered the Information Systems field. These tools allow researchers to uncover cognitive and emotional responses to the Internet. Since the information systems field partly exists to understand how individuals process information when interacting with technology, a deeper understanding of the cognitive and emotional underpinnings when using technology is warranted.

This minitrack provides a venue for research that examines cognitive and emotional correlates of the Internet and Digital Economy. Specifically, this minitrack calls for research that utilizes neuroimaging methodologies that augment our understanding of the Internet. Research examining vision, the neural system, and other "deep" inspections of how humans process, transmit, and reach shared understanding of information through the use of technology will be considered. The goal of this minitrack is to advance the use of these methodologies in IS by bringing together researchers to share their work and insights.

This minitrack calls for insightful papers examining cognition and emotion on the Internet. Following is a list of possible topics for prospective papers for this minitrack and is not meant to be exhaustive:

- The examination of cognition or emotion on the Internet using Electroencephalography (EEG), functional Magnetic Resonance Imaging (fMRI), eye-tracking, or psychophysiology measures (e.g., electrodermal response, heart rate).

- Human-Computer Interaction (HCI) on the Internet or in the Digital Economy

- Augmented Cognition using Internet platforms

- Theory-driven cognitive neuroscience in information systems

- Using the brain and technology to enhance individual and team performance on the Internet

- Psychophysiological responses to online advertising, shopping, or other interactions with the Digital Economy 